\title{
Diagnosis and Treatment of B Non-Hodgkin Lymphoma with Systems Biology Approaches
}

\author{
Ali Salari ${ }^{1 *}$, Zahra Zanganeh, ${ }^{2}$ Mansour Ebrahimi ${ }^{3}$ \\ ${ }^{1}$ Young Researcher and Elite Club, Borujerd Branch, Islamic Azad University, Borujerd, Iran. \\ ${ }^{2}$ Department of Basic Sciences, Biology group, Islamic Azad University of Hamedan, Hamedan, Iran. \\ ${ }^{3}$ Department of Biology, School of Basic Sciences and Bioinformatics Research Group, University of Qom, Qom, Iran.
}

\section{Received: 5 Feb 2016}

Revised : 11 Mar 2016

Accepted: 28 Mar 2016

Corresponding Author: Ali Salari

Young Researcher and Elite Club, Borujerd Branch, Islamic Azad

University, Borujerd, Iran

Phone: +989384922692

E-mail: Asalari1365@gmail.com

\begin{abstract}
Background: Lymphomas are solid tumors of immune system and Non-Hodgkin Lymphomas (NHL) are the most prevalent lymphomas; with wide ranges of histological and clinical features, it is so difficult to identify them. Herein, various bioinformatics tools (such as gene differential expressions, epigenetics and protein analysis) are employed to find a new treatment approach for NHL based on gene expression variation between classic Hodgkin and B NHL.

Materials and Methods: Microarray libraries GSE20011 were downloaded from NCBI database and analyzed with GEO2R software, then differential expression genes were analyzed by four databases (DAVID, Wikipathways, BioCarta and KEGG databases). Kinase, transcription factor, microRNA analysis and proteinprotein interaction network performed by $\mathrm{X} 2 \mathrm{~K}$, ChEA, microRNA TargetScan and Genes2Networks software respectively. Finally, drug target was identified and carried out by Drug Pair Seeker and Connectivity MAP databases.

Results: The results showed GATA2 Transcription Factor (TF) up-regulates genes while Sox2 down-regulates them.

Conclusion: Functional analysis of up-regulated genes showed high activation in B cell receptor signaling pathway while programmed cell death and apoptosis program were noted in down-regulated genes. Drug discovery facilities revealed that Verteporfin drug induces down-regulated genes while Prochlorperazine represses up-regulated genes. Three microRNA34al34c and miR-449 repressed up-regulated gene networks. The findings pave the way toward B-NHL therapy with 34a/b and miR-449 microRNAs and Prochlorperazine / Verteporfin drugs.
\end{abstract}

Keywords: B non-Hodgkin lymphoma; Enrichment analysis; Systems Biology

Please cite this article as: Salari A, Zanganeh Z, Ebrahimi M. Diagnosis and Treatment of B Non-Hodgkin Lymphoma with Systems Biology Approaches. Res Mol Med. 2016; 4 (2):

\section{Introduction}

Lymphomas are solid tumors of immune system and $90 \%$ of them have been diagnosed as Non-Hodgkin Lymphomas (NHLs), so far (1). The diagnosis of NHL is difficult due to the fact that their the histological appearances and clinical features are divers (1). The disease usually occurs in patients in late fifties and older. It is the fifth most frequently diagnosed cancer in the UK (1) as well as in the globe. Near 210000 deaths from NHL were reported between 1990 and 2010. (2).

Demographic data shows the incidence of the agestandardized disease in some countries such as UK, Scotland and Wales has increased by $35 \%$ in 30 years
(1988-2007) (3-5). In USA, 3.7\% yearly increase in just 15 years (1992-2007) has been reported and the same trend has also been documented in other countries such as Brazil, India, Japan, Singapore, and Western Europe (6-8). Immunosuppression has been blamed as the most well-established risk factor for the development of NHL; therefore, higher incidence of the disease has been reported in HIV positive patients. Those who receive immunosuppressive medicine (such as organ-transplant recipients, patients who have had high-dose chemotherapy with stem-cell transplantation, and those with inherited immunodeficiency syndromes or autoimmune disease) 
have also shown higher cases of NHL. Inhibition of immune system by infectious agents, or induction of chronic inflammatory diseases facilitates the incidence of lymphoma. Some infections (such as the Epstein-Barr virus or Helicobacter pylori) are risk factors for lymphoid tissue lymphoma. It has been shown that some specific lymphomas are associated with some microorganisms such as hepatitis $\mathrm{C}$ virus, Borrelia burgdorferi with cutaneous mucosaassociated lymphoid tissue lymphoma, and Chlamydia psittaci with ocular adnexal lymphoma (9).

Bioinformatics represents a new field at the interface of the twentieth-century revolutions in molecular biology and computers. A focus of this new discipline is the use of computer databases and computer algorithms to analyze huge available data on protein and genes (10). Extracting sense of the enorrmous quantities of biological data is a major challenge in bioinformatics era (11). Microarray techniques classified in high-throughput techniques and the result of microarrays revealed Differential Expression Genes (DEGs) between different stages of disease. These methods may enable a better understanding of the disease mechanisms (12-14). In this study, systems biology methods and bioinformatics tools have been employed to better understanding of B Non-Hodgkin Lymphoma (BNHL) mechanism and to predict new candidate approach of diagnosis and treatment.

\section{Materials and Methods}

Microarray analyses

Microarray data (GSE20011 dataset) was downloaded from National Center Biotechnology Institute (NCBI), Gene Expression Omnibus (GEO) database. Data was analyzed by GEO2R (www.ncbi.nlm.nih.gov/geo/geo 2r) tools and Non-Hodgkin lymphoma (NHL) and Classic Hodgkin lymphoma (CHL) were compared to identify differential expression genes (15).

Differential expression Genes functional analysis was performed with two DAVID and BioCarta database for differential expression genes (DEGs) (16-18) and their molecular functional properties were analyzed by GO Molecular Function via Enrichr (www.amp.pharm.mssm.edu/Enrichr) web site (16).

Disease analysis

Differential expression genes were applied to OMIM disease database by Enrichr web tools (16).

\section{Cellular Component Analysis}

To identify the DEGs position in the cell structure. We analyzed data with Gene Ontology Cellular Component database (16).

Protein Analysis

Differential expression genes analyses were performed by ChEA database to find Transcription factors (TFs) on the promoter and upstream of promoter sites (16, $19)$; and to find intermediate proteins $(19,21)$ and protein kinase $(19,20)$.

\section{Pathways}

Signaling pathway was run by yEd (Version 3.10.2) graph editorsoftware (22) to understand the underlying mechanism of protein - protein interactions based on TFs, intermediate proteins and protein kinases.

\section{Drug Prediction}

The pathways showed many detail of protein mechanisms and possible effective drugs predicted by DPS (drugs pair seeker) and CMAP (Connectivity MAP) databases based on discovered pathways and differential expression genes $(16,19,20)$.

\section{MicroRNAs Prediction}

We predicted both microRNAs working on suppress signaling pathways and up regulated genes by TargetScan microRNAs $(20,23)$.

\section{Results}

Microarrays analysis

The result showed the expression of 548 genes (371 up regulated and 177 down regulated) changed significantly in analyzed datasets.

Table 1. Functional analysis of DEGs.The first column is the function of URGs and the best function was shown B cell receptor signaling pathway and lymphocyte activation, whereas the second column is the function of down-regulated genes and the best function of DEGs was reported in programmed cell death, Cell death and Regulation of apoptosis.

\begin{tabular}{ll}
\hline Function of up-regulated genes & Function of down-regulated genes \\
\hline B cell receptor signaling pathway & Programmed cell death \\
Lymphocyte activation & Cell death \\
Leukocyte activation & Death \\
Pleckstrin homology & Regulation of apoptosis \\
B cell activation & Regulation of programmed cell death \\
Immune response-activating cell surface receptor Signaling pathway & Regulation of cell death \\
Immune response-activating cell surface receptor Signaling pathway & BzIp transcription factor/bzIp-1 \\
Immune response-activating signal transduction & BRLZ \\
\hline
\end{tabular}


Function Analysis

The result of function analysis showed in upregulated genes B cell receptor signaling pathway and lymphocyte activation mechanisms were dominant, whereas in down-regulated programmed cell death and cell death mechanisms were no table (Table 1).

Molecular function of DEGs showed that receptor protein signaling, iron ion binding and heme binding functions were the most affected functions in upregulated genes; whereas in down-regulated genes they were transcription regulator, RNA polymerase 2 transcription factor and transcription factor binding (Supplemental Table 1).

Disease analysis

DEGs were analyzed with OMIM disease database, and the up-regulated genes werereported in Ichthyosis, Systemic-lupus-erythematosus and, Immunodeficiency diseases, whereas among significantly down-regulated genes, the reported diseases were Charcot-marie-toothdiseas, Asthma and Ectodermal-dysplasia (Supplemental Table 2).

Table 2. Promoter analysis of up-and down-regulated genes showed GATA2 and SOX2 as the best TFs in up- and downregulated genes respectively.

\begin{tabular}{llll}
\hline TF & Target & p-value & Combined score \\
\cline { 1 - 2 } \multicolumn{1}{l}{ Up-regulated } & & & \\
\cline { 1 - 2 } GATA2 & 51 & $1.45 \mathrm{E}-11$ & 47.22389 \\
GATA1 & 28 & $1.43 \mathrm{E}-09$ & 46.79499 \\
FOXA2 & 68 & $5.36 \mathrm{E}-17$ & 43.17111 \\
SOX2 & 53 & $1.27 \mathrm{E}-11$ & 36.61537 \\
CTNNB1 & 29 & $5.31 \mathrm{E}-10$ & 32.76541 \\
AR & 65 & $5.05 \mathrm{E}-12$ & 31.27659 \\
LMO2 & 41 & $8.55 \mathrm{E}-11$ & 30.81776 \\
TBX3 & 25 & $5.65 \mathrm{E}-07$ & 23.82473 \\
MITF & 94 & $3.93 \mathrm{E}-15$ & 23.74851 \\
POU3F2 & 35 & $6.29 \mathrm{E}-08$ & 23.2882 \\
\cline { 1 - 1 } Down- regulated & & & \\
\cline { 1 - 1 } SOX2 & 49 & $1.03 \mathrm{E}-19$ & 68.00847 \\
STAT3 & 24 & $1.60 \mathrm{E}-05$ & 44.98689 \\
TCFAP2C & 41 & $2.51 \mathrm{E}-13$ & 41.21353 \\
GATA1 & 43 & $4.79 \mathrm{E}-15$ & 36.25311 \\
MYB & 21 & $4.35 \mathrm{E}-10$ & 35.45965 \\
HNF4A & 76 & $2.00 \mathrm{E}-20$ & 33.23756 \\
SALL4 & 19 & $5.63 \mathrm{E}-08$ & 32.7681 \\
FOXA2 & 45 & $2.04 \mathrm{E}-14$ & 32.76121 \\
KLF4 & 28 & $6.10 \mathrm{E}-10$ & 32.29828 \\
\cline { 1 - 1 } E2F4 & 44 & $1.30 \mathrm{E}-13$ & 30.43986 \\
\hline
\end{tabular}

rmm.mazums.ac.ir

\section{Cellular Component}

Membrane and nuclear membrane were two components of cells that significantly were employed by up-regulated genes, whereas in down-regulated genes cytoplasmic membrane-bounded vesicle was the most activated one (Supplemental Table 3).

Table 3. Specific protein kinase extracted from intermediate proteins that were extracted from DEGs are shown. The first column showed kinases names and the second column showed combine of three P-value, Z-score and Adjusted p-value scores which is called combined scores. The best kinase of URGs is LYN and it is not reported in DRGs and AURKA kinase is reported in DRGs and is not reported in URGs.

\begin{tabular}{ll}
\hline Term & Combined score \\
\hline Specific Protein Kinase of & \\
\hline URGS & -3.129911218 \\
BTK & -3.975363013 \\
PRKCG & -5.023054237 \\
ERBB4 & -7.104337302 \\
MST1R & -8.21716952 \\
MAP3K5 & -8.754972947 \\
ERBB3 & -9.130840177 \\
KIT & -9.414200374 \\
IKBKE & -9.695227483 \\
TBK1 & -9.765171072 \\
\hline Specific Protein Kinase of & \\
DRGs & \\
\hline AURKA & 5.970405374 \\
ATR & -0.894446961 \\
CHEK2 & -2.099157995 \\
PLK1 & -6.177234754 \\
AURKB & -9.663050797 \\
CAMK2B & -10.30617005 \\
SMG1 & -27.51413737 \\
TP53RK & -30.51656238 \\
HIPK1 & -32.12118479 \\
TTK & -34.99793532 \\
PKMYT1 & -37.34807626 \\
BRD2 & \\
\hline & \\
\hline
\end{tabular}

Protein analysis

Transcription factor (TF), intermediate protein and protein kinase analysis of both URGs and DRGs confirmed that two signaling pathways were involved in the process. TFs analysis of DRGs reported that GATA2, GATA1 and FOXA2 were the best TFs binding to URGs and SOX2, STAT3 and TCFAP2C were the best $T F S$ in down-regulated genes list (Table 2). 
When two TFs lists of URGs and DRGs were compared, the overall TFs were the same except for HTT, IKZF1, ZNF652, HOXD13, SALL1, CHD7, CIITA, BP1 in URGs and TRP63, DMRT1, NR4A2, IRF1 in DRGs.

TFs of URGs and DRGs were analyzed with intermediate proteins analysis and results showed the 79 and 40 intermediate proteins respectively. The intermediate proteins could be helping the TFs to be active in the cells.

The protein kinases analyses from intermediate proteins pointed out 219 protein kinases of intermediate protein from URGs; the best scores were gained by MAPK1, MAPK3, AKT1 respectively, whereas 155 kinases were in DRGs intermediate proteins and the best scores were assigned to MAPK1, MAPK3 and HIPK2 respectively. Two types of kinases were compared and it was found that overall kinases were the same except for 12 kinases of DRGs and 76 kinases from URGs; the best scores were gained by LY BTK, PRKCG in URGs whereas AURKA, ATR, CHEK2 gained the best scores in DRG (Table 3).

\section{Pathways}

Pathways among TFs, intermediate proteins and protein kinases were used to understand the mechanisms of DEG up-regulated or down-regulated genes; and to find the protein hubs to diagnose their therapeutic target candidates. Results showed Androgen Receptor (AR) was the hub protein of URG via 25 direct and 3 indirect interactions (Figure1).

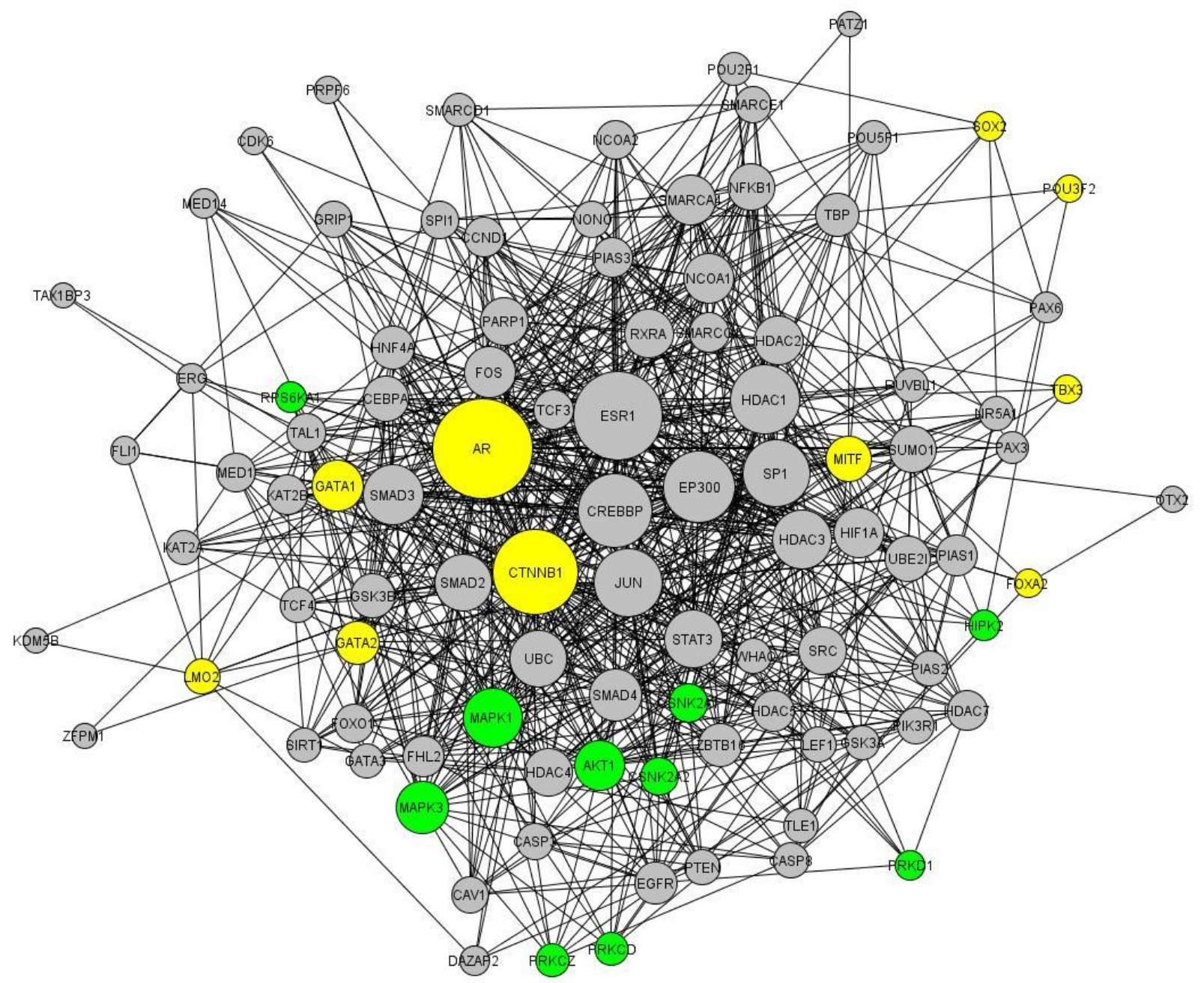

Figure 1. Pathway created of 3 proteins founded in this study as TFs and intermediate protein and kinases. After finding three types of protein from DEGs, we made the above pathway. The above pathway has involved 99 nodes and 812 edges that mean internal interaction among proteins. 79 intermediate proteins are shown with Light gray and $10 \mathrm{TFs}$ with yellow and 10 kinases with green.

Core protein of pathways showed Androgen Receptor (AR) TFs with 25 direct edges and 3 indirect edges. 
Hubs of protein pathway of DRG showed that signal transducer and activator of transcription 3 (STAT3) with 29 direct interactions were the main proteins (Figure2).

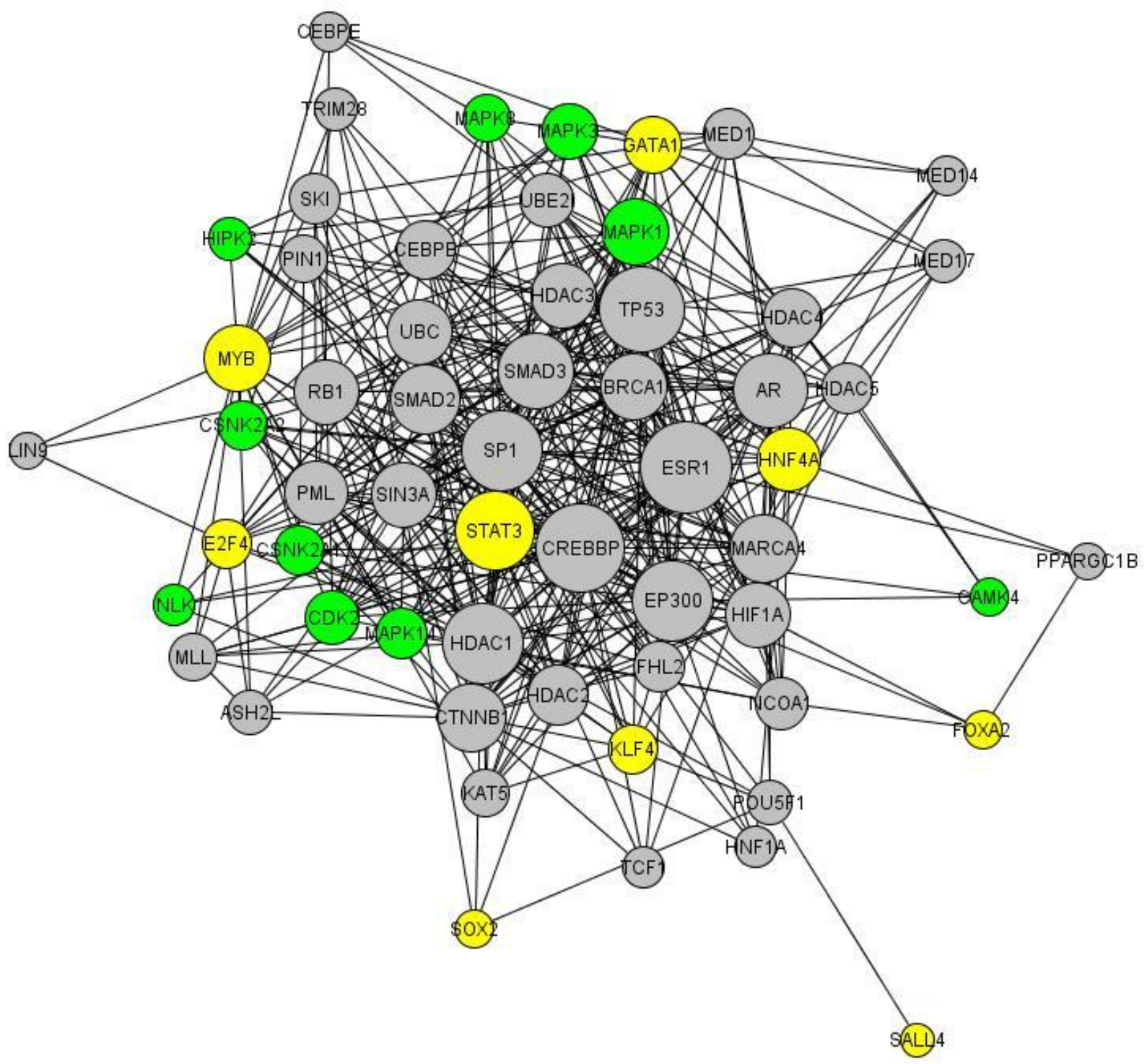

Figure 2. Pathway created of 3 proteins founded in this study as TFs and intermediate protein and kinases. After finding three types of protein from DEGs, we made the above pathway. The above pathway has involved 60 nodes and 441 edges that mean internal interaction among the proteins. 40 intermediate proteins colored with light-gray and $10 \mathrm{TFs}$ with white and 10 kinases with dark gray.

Core protein of pathways showed Signal Transducer and Activator of Transcription 3(STAT3) TFs with 29 direct edges and Mitogen-Activated Protein Kinases1 (MAPK1) of kinase with 21 edges and Estrogen Receptor 1 (ESR1) via 37 edges reported.

\section{Drug Prediction}

Drug prediction was performed to both pathways and both URGs and DRGs to find the suitable drug candidate to BNHL therapy and prevention by drug prediction. The first predicted drugs to suppress URGs was rochlorperazine $(0.0000$ Molar (M) dosage for 6 hours) and BCB000040 (0.00001 M dosage for 6 hours); the drugs predicted to induce
DRGs as Verteporfin $(0.0000028 \mathrm{M}$ dosage for 6 hours) and Adenosine phosphate $(0.000011 \mathrm{M}$ dosage for 6 hours) (Table 4).

The second predicted drugs to revers proteins pathway were Trichostatin A (via different dosage), Nordihydroguaiaretic acid $(0.000001 \mathrm{M}$ dosage for 6 hours) (Supplemental Table 4). 
Table 4. Drugs predicted of DEGs. The first column showed the drugs to suppress the up-regulated genes (URGs) and the second column reported drugs to induce the down-regulated genes (DRGs).

\begin{tabular}{ll}
\hline Suppresser Drugs to URGs & Inducer Drugs to DRGs \\
\hline Prochlorperazine-2675 & Verteporfin-6817 \\
BCB000040-7559 & Verteporfin-3556 \\
Trichostatin A-1175 & Adenosine phosphate-5359 \\
Vorinostat-2680 & Oxprenolol-5871 \\
Vorinostat-1161 & Pregnenolone-4218 \\
Carcinine-1305 & Atovaquone-4786 \\
Guanabenz-4642 & Altizide-2527 \\
Ciclopirox-2456 & Capsaicin-3034 \\
Equilin-5620 & Lansoprazole-2967 \\
Sodium phenylbutyrate-502 & Palmatine-4957 \\
\hline
\end{tabular}

MicroRNA Prediction: MicroRNAs predicted to suppress URGs and proteins pathway, the results showed that the miR-34A, miR-34C, and miR-449 as the best suppressor for URGs. Whereas the miR199A and miR-199B were two microRNAs which act on proteins pathway (Figure 1) (Table 5).

Table 5. MicroRNAs has predicted to suppress the URGs and proteins pathway, the first part of the table report which microRNAs were predicted to suppress the URGs and the second part showed which microRNAs to suppress the proteins pathway. The first column showed the microRNA names and the second column report the score value (which P-value less than 0.05). The best microRNAs to suppress the URGs reported in miR-34A, miR$34 \mathrm{C}$ and mir-449, also the best of second part which suppress the proteins pathway reported in miR-199A and miR-199B.

\begin{tabular}{ll}
\hline Term & P-value \\
\hline Revers up-regulated genes & 0.0047596 \\
miR-34A, miR-34C, miR-449 & 0.0077706 \\
miR-219 & 0.008243 \\
\hline Revers protein pathway & \\
\hline miR-199A,miR-199B & $1.61 \mathrm{E}-04$ \\
miR-18A,miR-18B & 0.0059645 \\
miR-1,miR-206 & 0.0079639 \\
miR-524 & 0.0083552 \\
\hline
\end{tabular}

\section{Discussion}

Lymphoma is known as solid tumor in the immune system. Non-Hodgkin lymphoma makes up most cases of lymphoma and less than $10 \%$ of the cases are Hodgkin lymphoma (1). Diagnosis of nonHodgkin lymphoma is difficult because of histological appearances and clinical features (1). Herein, to understand the underlying mechanisms of
NHL and to find the best target elements to diagnose and treat it, we employed various bioinformatics and system biology tools to find the candidate proteins, genes and drugs. Microarray analysis was used to compare B non-Hodgkin lymphoma (Malignant form) and Classic-Hodgkin Lymphoma (Benign form). There were 371 URGs and 177 DRGs. Functional analysis revealed that DRGs were associated with cell death, while URGs were related to B cell receptor signaling pathways, lymphocyte activation and leukocyte activation (Table 1).

Molecular function analysis was performed in URGs. The results revealed genes have functioned in heme binding and calcium ion transmembrane transporter, whereas the results of molecular function analysis in DRGs are reported to function in MHC class I receptor in which is important in immune response mechanism.

Of ten first molecular functions (Supplemental table 1), four functions contribute to transcription mechanisms, for example the RNA polymerase II transcription factor activity.

The function of two genes SOCS1 and CDKN1B in the DRGs are reported in protein kinases inhibitor. Molecular function analysis was performed to determine specific protein kinase from DEGs (Table 3). The results of molecular function of seven protein kinases AURKA, ATR, CHEK2, PLK1, SMG1, TP53RK and HIPK1 in comparison with the ten first of kinase molecular functions (Supplemental table 1) are reported in transcription mechanisms.

URGs were analyzed by disease database, and the results showed those contribute to Ichthyosis disease as well as immunodeficiency disease (Supplemental table 2).

Cellular component analysis was performed to URGs. The results showed that the membrane component. The membrane component is the one of the important organ of cells stability and resistance. The URGs working found to membrane against CHL it is may a sign or a reason to increase the cells resistance and stability in malignant BNHL. (Supplemental table 3).

GATA2 TFs are reported as the importance TFs in URGs, and SOX2 showed the main TFs in the DRGs (Table 2), the specific TFs were not reported in DRGs but were reported in the URGs. So, HTT, IKZF1, ZNF652, HOXD13, SALL1, CHD7, CIITA, BP1 are the specific TFs for URGs whereas TRP63, DMRT1, NR4A2, IRF1 are the specific TFs for DRGs. Specific TFs may have the potential to be candidate for the diagnosis or treatment target.

In the protein kinase analysis, the best scores has been gained from Mitogen-Activated Protein Kinases1 (MAPK1) and the specific protein kinase was reported by LYN in the reported URGs. 
LYN is one of the Tyrosine kinases of src family and has been reported to have an important role in the Immunodeficiency disease like Asthma. Other LYN functions are also reported in the negative control of $\mathrm{B}$ receptor cells and are being important to remain the phenotype of acute myeloid leukemia (AML) and B cell lymohocytic leukemia (BCL) (23-25). Therefore, LYN protein kinase may have an important role in BNHL, and also may have potential to be use for diagnosis and treatment targeting. The best score of protein kinases of DRGs are reported for MitogenActivated Protein Kinases1 (MAPK1), but MAPK1 gained the best score of both URGs and DRGs so it is not a suitable candidate for any targeting.

In DRGs the best score of specific protein kinase was gained by Aurora Kinase A (AURKA).

Peaks of activity of AURKA are reported in G2 phase to M phase transition in the cell cycle (26). It's very important because it is working in the phase that the mutated cells are going to unlimited or without any proliferation control like cancer cells.

Seven protein kinases AURKA, ATR, CHEK2, PLK1, SMG1, TP53RK and HIPK1 are reported to phosphorylate the TP53 main tumor suppressor protein (27).

The finding revealed that the above seven protein kinases activate the TP53 by phosphorylating in BNHL cells. Hence, AURKA may play an important role in BNHL. In other words, functions of DRGs were reported on the Cell death and Apoptosis and functions of seven protein kinases of DRGs were reported to phosphorylate TP53 which is one of the main proteins of cell death function (28-31). This finding can explain the cell death mechanisms that there are two paths to activate the cell death programs. The first one which is direct path includes seven protein kinases and leading to cell death program by activating TP53. The second path is indirect path which activates the DRGs, resulting in activating cell death program.

In this study reported by the first time both hub proteins may be the potential candidates for the diagnosis and treatment targeting.

Drug prediction revealed that the Prochlorperazine with 0.00001 Molar (M) dosage for 6 hours and BCB000040 with $0.00001 \mathrm{M}$ dosage for 6 hours suppress the URGs. Also Verteporfin with 0.0000028 $\mathrm{M}$ dosage for 6 hours and Adenosine phosphate $0.000011 \mathrm{M}$ dosage for 6 hours are predicted to induce DRGs (Table 4).

Other results showed that the Trichostatin A via different dosage and Nordihydroguaiaretic acid with $0.000001 \mathrm{M}$ dosage for 6 hours are predicted to suppress the proteins pathway (Figure 1). Afterward the results obtained that the Resveratrol with $0.0000176 \mathrm{M}$ dosage for 6 hours induces the proteins pathway Figure 2 (Supplemental Table 4). This finding is reported for the first time to utilize the candidate drugs for new therapeutic approaches.

Mir-34A and miR-34C and miR-449 are predicted to suppress the URGs with the therapeutic approaches. The results also showed that miR-199A and 199B suppress the protein pathway (Figure 1). This finding is reported for the first time and it could be utilized for molecular therapy (Table 5).

The suggested drugs are utilized together which are predicted to reverse URGs and to induce DRGs and the suppressed protein pathways (Figure 1). It is also possible to improve and optimize the therapeutics candidate method has been suggested in this study.

The reported candidate protein molecules and drugs in this study need to be validated with molecular approaches in the experimental laboratory.

\section{Conclusion}

In summary, this study suggests GATA2 and SOX2 as the two importance markers in BNHL. The findings also pave the way toward B-NHL therapy with miR-34a/b and miR-449 microRNAs and Prochlorperazine with $0.00001 \mathrm{M}$ dosage for 6 hours and Verteporfin with $0.0000028 \mathrm{M}$ dosage for 6 hours drugs.

\section{Acknowledgements}

The authors greatly appreciate the Systems Biology Lab of Salari Behavior and Cognitive Disorders Institute, Alborz, Iran, and Salar OMIM Company for its valuable supports.

\section{Author contributions}

SA designed and performed experiments analyzed data and wrote the paper, $\mathrm{ZZ}$ performed experiments and EM wrote the paper.

\section{Conflict of Interest}

The authors declare that they have no conflict of interest in this article.

\section{Support/Funding}

This study supported by Salar OMIM Companies (SOC) and Salari Institute of Cognitive and Behavioral Disorders (SICBD).

\section{References}

1. Shankland KR, Armitage JO, Hancock BW. Non-Hodgkin lymphoma. Lancet. 2012; 380(9844):848-57. PMID: 22835603.

2. Lozano R, Naghavi M, Foreman K, Lim S, Shibuya K, Aboyans $\mathrm{V}$, et al. Global and regional mortality from 235 causes of death for 20 age groups in 1990 and 2010: a systematic analysis for the Global Burden of Disease Study 2010. Lancet. 2012; 380(9859):2095-128. PMID: 23245604.

3. Parkin DM, Boyd L, Walker LC. 16. The fraction of cancer 
attributable to lifestyle and environmental factors in the UK in 2010: Summary and conclusions. Br J Cancer. 2011; 105(Suppl 2):S77-S81. PMID: 22158327

4. Statistics Of, N. "Cancer Statistics registrations: Registrations of cancer diagnosed in 2008, England." Office for National Statistics, National Statistics London (2010).

5. Shack L, Jordan C, Thomson CS, Mak V, Møller H, UK Association of Cancer Registries. Variation in incidence of breast, lung and cervical cancer and malignant melanoma of skin by socioeconomic group in England. BMC Cancer. 2008;8:271. PMID: 18822122.

6. Morton LM, Wang SS, Devesa SS, Hartge P, Weisenburger DD, Linet MS. Lymphoma incidence patterns by WHO subtype in the United States, 1992-2001. Blood. 2006; 107(1):265-276. PMID: 16150940

7. Sant M., Allemani C., Tereanu C., De Angelis R., Capocaccia R., Visser O., Marcos-Gragera R., Maynadie M., Simonetti A., Lutz J.M., Berrino F. Incidence of hematologic malignancies in Europe by morphologic subtype: results of the HAEMACARE project. Blood. 2010; 116:3724-3734. PMID: 20664057.

8. Naresh KN, Srinivas V, Soman CS. Distribution of various subtypes of non-Hodgkin's lymphoma in India: a study of 2773 lymphomas using R.E.A.L. and WHO Classifi cations. Ann Oncol 2000; 11 (suppl 1): 63-67. PMID: 10707782

9. Ambinder, RF. Infectious etiology of lymphoma. in: JO Armitage, PM Mauch, NL Harris, B Coiffier, R Dalla-Favera (Eds.) Non-Hodgkin lymphomas. 2nd edn. Wolters Kluwer and Lippincott Williams and Wilkins, Philadelphia, PA; 2010: 83-101

10. Pevsner J. Bioinformatics and Functional Genomics: Second Edition. John Wiley and Sons, 2009. 951 p. Available from, DOI: 10.1002/9780470451496

11. Salari Ali RSM, Ebrahimi Mansour. Designing prediction models to determine the structure of Stearoyl-acyl carrier protein desaturase 1. Qom Univ Med Sci J. 2015; 9(8):13-20.

12. Kierzek R, Turner DH, Kierzek E. Microarrays for identifying binding sites and probing structure of RNAs. Nucleic Acids Res. 2015; 43(1):1-12. PMID: 25505162.

13. Yoo SM, Choi JH, Lee SY, Yoo NC. Applications of DNA microarray in disease diagnostics. J Microbiol Biotechnol. 2009; 19:635-46. PMID: 19652509.

14. Kierzek R, Turner DH, Kierzek E. Microarrays for identifying binding sites and probing structure of RNAs. Nucleic Acids Res. 2015; 43(1):1-12. PMID: 25505162.

15. Barrett T, Wilhite SE, Ledoux $\mathrm{P}$, Evangelista C, Kim IF, Tomashevsky $\mathrm{M}$, et al. NCBI GEO: archive for functional genomics data sets--update. Nucleic Acids Res. 2013; 41(Database issue):D991-5. PMID: 23193258.

16. Chen EY, Tan CM, Kou Y, Duan Q, Wang Z, Meirelles GV, et al. Enrichr: interactive and collaborative HTML5 gene list enrichment analysis tool. BMC Bioinformatics. 2013; 14:128. PMID: 23586463

17. Huang DW, Sherman BT, Tan Q, Kir J, Liu D, Bryant D, et al. DAVID Bioinformatics Resources: expanded annotation database and novel algorithms to better extract biology from large gene lists. Nucleic Acids Res. 2007; 35(Web Server issue):W169-75. PMID: 17576678
18. Huang da W, Sherman BT, Lempicki RA. Systematic and integrative analysis of large gene lists using DAVID bioinformatics resources. Nat Protc. 2009; 4(1):44-57. PMID: 19131956.

19. Chen EY, Xu H, Gordonov S, Lim MP, Perkins MH, Ma'ayan A. Expression2Kinases: mRNA profiling linked to multiple upstream regulatory layers. Bioinformatics. 2012; 28(1):105-11. PMID: 22080467

20. Salari A, Salarian F. Prediction of New Diagnostic and Therapeutic Approaches in Pulmonary Arterial Hypertension by Systems Biology Metho. Qom Univ Med Sci J. 2016; 10 (5):9-20

21. Berger SI, Posner JM, Ma'ayan A. Genes2Networks: connecting lists of gene symbols using mammalian protein interactions databases. BMC Bioinformatics. 2007; 8:372. PMID: 17916244

22. Raza S, Robertson KA, Lacaze PA, Page D, Enright AJ, Ghazal P, et al. A logic-based diagram of signalling pathways central to macrophage activation. BMC systems biology. 2008; 2:36. PMID: 18433497

23. Maziere P, Enright AJ. Prediction of microRNA targets. Drug Discov Today. 2007; 12(11-12):452-8. PMID: 17532529.

24. Ingley E. Functions of the Lyn tyrosine kinase in health and disease. Cell communication and signaling: CCS. 2012; 10(1):21. PMID: 22805580

25. Pillai S, Netravali IA, Cariappa A, Mattoo H. Siglecs and Immune Regulation. Annu Rev Immunol. 2012; 30:357-392. PMID: 22224769

26. Brauninger A, Renne C, Willenbrock K, Martin-Subero JI, Hinsch N, Tiacci E, et al. [Global gene expression analysis and novel signalling pathways in Hodgkin lymphoma]. Verh Dtsch Ges Pathol. 2006; 90:136-41. PMID: 17867590.

27. Mahen R, Venkitaraman AR. Pattern formation in centrosome assembly. Curr Opin Cell Biol. 2012; 24(1):14-23. PMID: 22245706

28. Kuppers R, Duhrsen U, Hansmann ML. Pathogenesis, diagnosis, and treatment of composite lymphomas. Lancet Oncol. 2014; 15(10):e435-46. PMID: 25186047.

29. Cox DG, Hankinson SE, Hunter DJ. Polymorphisms of the AURKA (STK15/Aurora Kinase) Gene and Breast Cancer Risk (United States). Cancer Causes Control: CCC. 2006; 17(1):81-3. PMID: 16411056.

30. Hainaut P, Olivier M. TP53 mutations in human cancers: functional selection and impact on cancer prognosis and outcomes. Oncogene. 2007; 26(15):2157-65. PMID: 17401424.

31. Petitjean A, Mathe E, Kato S, Ishioka C, Tavtigian SV, Hainaut P, Olivier M. Impact of mutant p53 functional properties on TP53 mutation patterns and tumor phenotype: lessons from recent developments in the IARC TP53 database. Hum Mutat. 2007; 28:622-629. PMID: 17311302.

32. Pinto EM, Ribeiro RC, Li J, et al. An identical, complex TP53 mutation arising independently in two unrelated families with diverse cancer profiles: the complexity of interpreting cancer risk in carriers. Oncogenesis. 2012; 1(2):e1-. PMID: 23552518. 